\title{
Prevention of Ventilator-Associated Pneumonia: Zero-VAP Implementation: Review Article
}

\author{
Dalia A. Abdulrahman, Mohammed Mahmoud Romih, Eman Mustafa Fathy El Attar \\ Department of Pediatrics, Faculty of Medicine, Zagazig University, Egypt \\ *Corresponding author: Eman Mustafa Fathy El Attar, E-mail: ema.elattar@ gmail.com
}

\begin{abstract}
Background: Mechanical ventilation via an endotracheal tube (ETT) or tracheostomy can lead to a condition known as ventilator associated pneumonia (VAP). Up to $20 \%$ of all nosocomial infections in pediatric care facilities following blood stream infections are most commonly brought on by Ventilator Associated Pneumonia (VAP). Pediatric intensive care units (PICUs) and neonatal intensive care units (NICUs) had very different rates of VAP infections per 1000 ventilator days. Patients who have VAP are more likely to have a longer stay in the hospital and a higher rate of morbidity and mortality. If implemented, "Zero VAP" was found to be an all-encompassing strategy for prevention of higher rates of VAP in Spain. This strategy could be widely used for more prevention of VAP globally.

Objective: To evaluate the role of the Spanish "Zero- VAP" bundle in reduction of VAP in PICU. Conclusion: ZeroVAP compromises of many levels among health working personnel and system, it gives a clear description of steps, approach, preventive actions to minimize incidence of VAP among ICUs that could be used globally.
\end{abstract}

Keywords: Ventilator Associated Pneumonia, Pediatric intensive care units.

\section{INTRODUCTION}

Mechanically ventilated patients are more likely to develop ventilator associated pneumonia (VAP), which is a type among nosocomial pneumonia that usually happens 48-72 hours after the patient has received mechanical ventilation. VAP is one of the most prevalent hospital-acquired infections in mechanically ventilated patients, and it is related with increased morbidity and mortality in these patients ${ }^{(\mathbf{1})}$.

Ventilator is a device that delivers oxygen to a patient's lungs via a tube inserted into his or her mouth, nose, or a hole in the front of the patient's neck. It is possible that bacteria will enter the patient's lungs via the tube and cause an illness ${ }^{(1)}$. In order for VAP to occur, the oropharynx and stomach must be vaccinated. Typically, VAP is acquired through aspiration from the paranasal sinuses, nose or even stomach (2). To prevent contamination of the connection port, extreme care must be taken when putting an endotracheal tube into the mechanical ventilator. Contagious diseases spread more easily when people have poor personal hygiene. As a result of mechanical ventilation utilizing heated humidifiers, bacteria may proliferate ${ }^{(3)}$.

The aim of this review was to evaluate the role of the Spanish "Zero- VAP" bundle in reduction of VAP in PICU.

Preventing pediatric ventilator-associated pneumonia: In the neonatal intensive care units, newborns that are dangerously unwell and preterm are cared for. The majority of mechanically ventilated paediatric patients are seen in the PICU, and they are typically between the ages of one and 21 days or months. $10 \%$ to $20 \%$ of mechanically ventilated patients suffer with VAP. Risk factors for this condition must be considered ${ }^{(4)}$.

Steps for VAP prevention: Interprofessional VAP management is based on an understanding of VAP's occurrence, risk factors, and prevention methods. To keep tabs on VAP or infection rates, it is essential to use bundled care and performance measurements ${ }^{(5)}$.

Shared protocols:

Pediatric patients must be treated with caution because they aren't ready to remain intubated without sedation. Some protocols are not suitable for use with children of all ages. Adults may benefit from daily sedation-free trials and spontaneous breathing trials to prevent VAP, but children may be at greater risk of unintentional extubation if these trials are offered ${ }^{(6)}$.

Interprofessional workflow or device management solutions suggested by surveillance models might be tested to see if they reduce the VAP rate. The Centers for Disease Control and Prevention (CDC) provides examples of pneumonia flow diagrams. Patients' outcomes can be improved by including various members of the PICU team ${ }^{(7)}$.

VAP Guidelines 2021:

New Guidelines for Treatment of VAP:

Antibiotics are effective against several bacterial types of VAP. For treatment suggestions, it is critical to identify the exact infections involved. Sputum samples may be collected by doctors who then give broad-spectrum antibiotics or even a variety of broadspectrum antibiotics as they await the results of the lab tests ${ }^{(8)}$. When a ventilated patient develops pneumonia, healthcare providers should not presume that the ventilator is at blame. Even without proper suctioning, aspiration pneumonia is common in this population. Similar to viruses, fungal infections can also cause lung infections, especially in vulnerable groups like patients in the intensive care unit (ICU). This is especially true. Get the patient to cough out a sample of his or her sputum for testing ${ }^{(9)}$.

Bronchoscopy specimens were cultured quantitatively, whereas Drakulovic and his 
colleagues $^{(10)}$ used clinical criteria and nonquantitative cultures in their earlier work to diagnose VAP in their previous study. Like earlier research, this one had a much larger number of participants (n 14 221) than those that came before it. While, this research has made great strides in recent years, there are still certain drawbacks. Semirecumbency's efficacy can't be conclusively determined because the intervention group couldn't reach the target 458 backrest position in this trial. Another flaw in the study is the mistaken assumption that the control group had a lower incidence of VAP. On the basis of previous data from their own groups, they expected a $25 \% \mathrm{VAP}^{(\mathbf{1 0})}$.

Guidelines for the prevention of ventilatorassociated pneumonia and their implementation. The Spanish 'Zero-VAP'" bundle:

Zero-VAP is a collection of VAP prevention measures used in ICUs in Spain that are implemented simultaneously in multiple modes. Methods: They were initiated and supported by the Spanish Societies of Intensive Care Medicine and Nursing, the project is open to anybody interested in learning more about intensive care medicine and nursing. In addition to VAP prevention advice, the Zero-VAP Plan includes a complete patient safety protocol as well as a continuous online evaluation of the bundle's use over time. VAP episodes and participation indices are saved online in an Infection Surveillance Program of Spain called "ENVIN-HELICS" database, providing realtime data on the incidence of VAP in the local, regional, and national contexts ${ }^{(\mathbf{1 1})}$.

At a minimum, there should be less than 9 VAP events per 1000 days of mechanical ventilation if the guidelines are followed exactly. A total of 35 measures of preventive were initially selected at the outset. 7 "required" recommendations were developed by a group of experts using the grading of recommendations, assessment, development and evaluation working group approach (stringent hand cleanliness, cuff pressure control, chlorhexidine oral hygiene, semi-recumbent positioning, advocating techniques that safely avoid or reduce time on the ventilator, and discouraging scheduled replacements of ventilator circuits and humidifiers, as well as endotracheal tubes) and three extra actions that are strongly recommended: Antibiotics given intravenously for a brief time, selective cleaning of the digestive tract as well as aspirating subglottic fluid ${ }^{(11)}$.

Catheter-related bloodstream infections and VAP can be reduced by implementing "bundles" of effective measures rather than single interventions, according to one theory. In 2008, the Spanish Society of Intensive Care Medicine (SEMICYUC) initiated and implemented an extremely effective bacteraemia prevention package, dubbed "Zero Bacteraemia," with the backing of the World Health Organization and the Quality Assurance Agency of the Spanish Ministry of Health (QAA) ${ }^{(11)}$.
"Zero-VAP" was first presented at a national meeting, then at regional and local ones, where the preventative bundle, goals, and methods for implementation and control were all discussed. According to the "Zero-VAP" standards, which are an extension of ENVIN-HELICS, which collect epidemiological data from all patients admitted to participating ICUs from April 1st to June 30th every year since 1994, and does not require ethics committee approval. There were no requests from the Spanish Ministry of Health or regional healthcare authorities for ethics committees to review the project as a quality improvement program ${ }^{(\mathbf{1 2})}$.

The selection of preventative methods for ventilator-associated pneumonia:

Using existing clinical trials, guidelines, systematic reviews and meta-analyses as a foundation, 35 therapies were developed. Functional, mechanical, and pharmaceutical classifications were used to categorize them (Table 1). Teams of at least two task force members evaluated independently utilizing the grading of recommendations assessment, development, and evaluation working group methodology. Eight controversial interventions were included in the final report after a quantitative evaluation by the panel's 11 members, who took into account the standard of proof (Ten points) and safety (five points) that can be implemented in Spanish intensive care units (five points). The criteria for practicality and cost were utilized as recommended ${ }^{(13)}$.

Table (1): Methods of VAP preventive classification $^{(13)}$.

\section{Functional:}

1. A situation of partial recumbency.

2. Prior to beginning any airway management, use an alcohol-based gel or solution to keep hands clean.

3. A course on bronchial aspiration training.

4. Weaning and extubation evaluations, as well as a daily sedation vacation.

5. A wide range of weaning methods are readily available.

6. Tracheostomy performed too soon.

7. Mechanical ventilation without the need for surgery.

8. Infection and cross-contamination monitoring by microbiologists.

9. Before endotracheal suctioning, saline infusion.

10. Changing of Tube.

11. Intubation through the trachea (nasotracheal versus orotracheal).

12. Type of humidification for the airways. Choosing between a heated humidifier and a heatexchanger.

13. Physiotherapy.

14. PEEP of $5-8 \mathrm{cmH}_{2} \mathrm{O}$ versus $\mathrm{ZEEP}$ in patients with no lung damage.

15. Gastric residual amount and administration method in enteral feeding. A drug called prokinetics. 


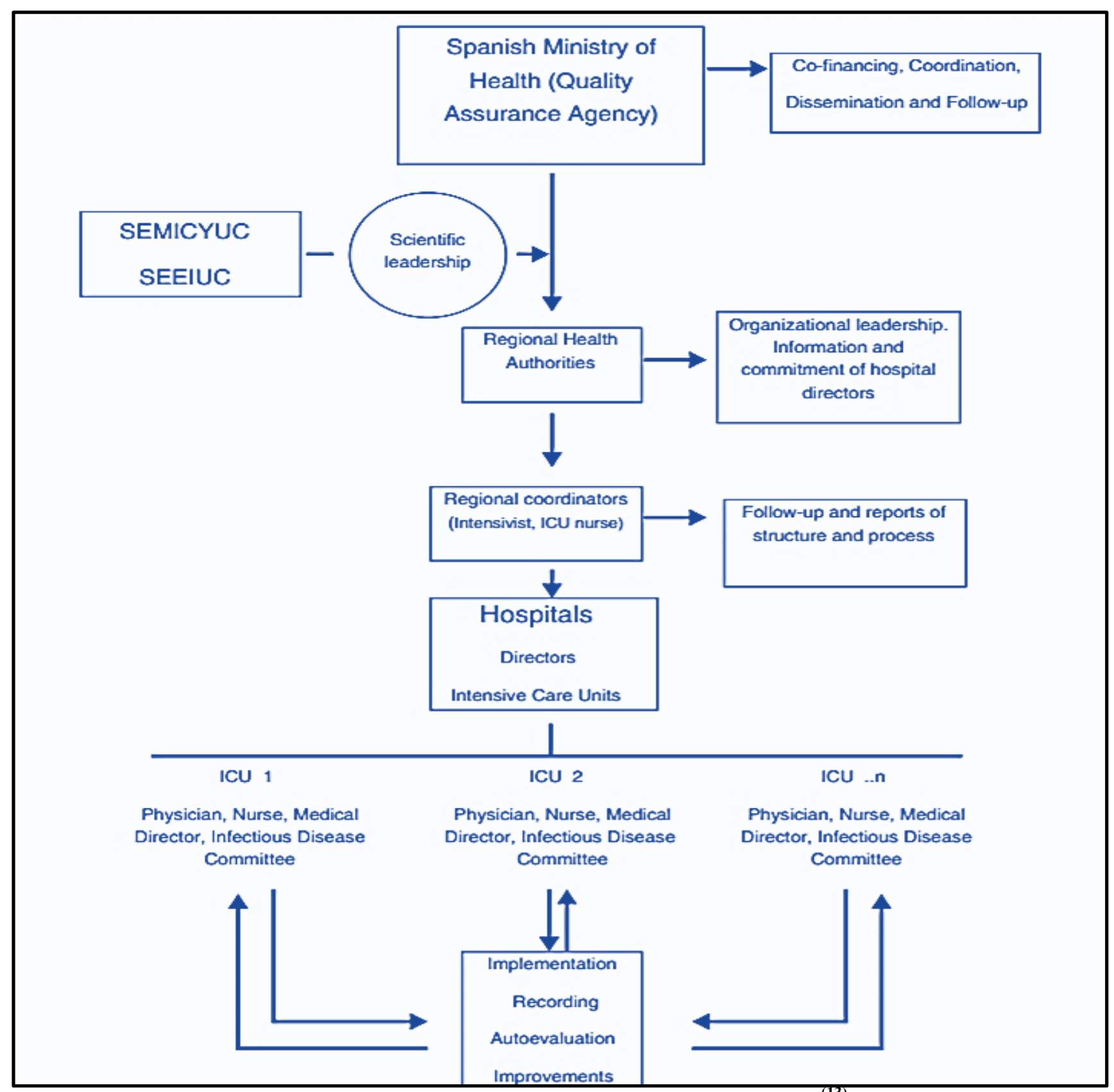

Figure (1): Zero VAP'” Project. Organizational scheme ${ }^{(13)}$.

Measures that must be taken as a matter of course:

1. Acquiring knowledge and skills in airway control (aspiration of bronchial secretions). Due to limitations in research design, it is unable to demonstrate a causal link between educational interventions and lower nosocomial infection rates in a comprehensive review that included 26 studies. Studies suggest that basic educational programs and therapeutic regimens, such as emphasizing strict hand hygiene, significantly reduce the prevalence of $\mathrm{VAP}^{(14)}$.

2. Alcohol-based hand sanitizers should be used prior to airway management. The prevalence of nosocomial infections and the spread of methicillin-resistant Staphylococcus aureus (MRSA) was shown to be lower in hospitals that promoted frequent hand washing. Preventative measures for VAP, such as hand-washing before coming to patient contact or usage of gloves were adopted in 2004. Standard clinical practice has now firmly established it as an essential component. Prior to and following the use of an artificial airway, the hands must be washed with alcohol solutions ${ }^{(\mathbf{1 5})}$.

3. Chlorhexidine solutions $(0.12--2 \%)$ should be used for oral hygiene every eight hours. Cuff pressure should be at least $20 \mathrm{cmH}_{2} \mathrm{O}$ prior to use. Nurse's aides, who are responsible for this operation in most intensive care units, should receive formal training ${ }^{(\mathbf{1 6})}$.

4. If the patient's cuff pressure falls below $20 \mathrm{cmH}_{2} \mathrm{O}$, they are at a higher risk of developing VAP. No evidence of a link was found in a recent study. The panel agreed that cuff pressure checks are a straightforward, low-cost, and conventional clinical technique that also helps to prevent excessively high pressures in the cuffs ${ }^{(17)}$.

5. Aspiration of contaminated stomach contents may be reduced by positioning the patient semi-recumbent. There is no evidence that it prevents VAPs in people who are unstable or have high intra-abdominal 
pressure. The results of a recent randomised experiment including 232 patients with tetanus were unfavorable, according to the study's authors. Patients receiving enteral nutrition should not be placed in a $0^{\circ}$ supine position ${ }^{(\mathbf{1 8})}$.

6. Avoiding or decreasing the duration of mechanical ventilation through promoting safe methods and protocols. VAP reductions are related with effective therapies targeted at preventing or reducing the length of endotracheal intubation. There should be NIMV procedures for acute exacerbations of COPD, for weaning, or to promote lower infusion dosages, or daily suspension of infusions, in order to be available (19)

7. Ventilator circuit adjustments that are part of a strategy could increase both costs and VAP risks. Heat-moisture exchangers (HME) should only be used in the most extreme instances where there is a high probability of an obstruction of the respiratory tract ${ }^{(20)}$.

\section{CONCLUSION}

Zero-VAP compromises of many levels among health working personnel and system, it gives a clear description of steps, approach, preventive actions to minimize incidence of VAP among ICUs that could be used globally.

\section{Financial support and sponsorship: Nil. Conflict of interest: Nil. \\ REFERENCES}

1. Branch-Elliman W, Wright S, Howell M (2015): Determining the ideal strategy for ventilator-associated pneumonia prevention. Cost-benefit analysis. American Journal of Respiratory and Critical Care Medicine, 192 (1): 57-63.

2. Rouzé A, Martin-Loeches I, Nseir S (2018): Airway devices in ventilator-associated pneumonia pathogenesis and prevention. Clinics in Chest Medicine, 39 (4): 775-783.

3. Mohanty D, Routray S, Mishra D et al. (2016): Ventilator associated pneumonia in a ICU of a tertiary care Hospital in India. IJCMR., 3 (4): 1046-9.

4. Iosifidis E, Pitsava G, Roilides E (2018): Ventilatorassociated pneumonia in neonates and children: a systematic analysis of diagnostic methods and prevention. Future Microbiology, 13 (12): 1431-1446.

5. Peña-López Y, Pujol M, Campins M et al. (2016): Implementing a care bundle approach reduces ventilator-associated pneumonia and delays ventilatorassociated tracheobronchitis in children: differences according to endotracheal or tracheostomy devices. International Journal of Infectious Diseases, 52: 43-4.

6. Vijay G, Mandal A, Sankar J et al. (2018): Ventilator associated pneumonia in pediatric intensive care unit: incidence, risk factors and etiological agents. The Indian Journal of Pediatrics, 85 (10): 861-866.

7. Gupta S, Boville B, Blanton $R$ et al. (2015): A multicenter prospective analysis of diagnosis, risk factors, and outcomes associated with pediatric ventilator-associated pneumonia. Pediatric Critical Care Medicine Society of Critical Care Medicine, 16 (3): 6573.

8. Nair G, Niederman M (2015): Ventilator-associated pneumonia: present understanding and ongoing debates. Intensive Care Medicine, 41 (1): 34-48.

9. Spalding M, Cripps M, Minshall C (2017): Ventilator-associated pneumonia: new definitions. Critical Care Clinics, 33 (2): 277-292.

10. Drakulovic M, Torres A, Bauer T et al. (1999): Supine body position as a risk factor for nosocomial pneumonia in mechanically ventilated patients: a randomised trial. Lancet, 354:1851-1858.

11. Álvarez-Lerma F, Palomar-Martínez M, SánchezGarcía M et al. (2018): Prevention of ventilatorassociated pneumonia: the multimodal approach of the Spanish ICU "Pneumonia Zero" program. Critical Care Medicine, 46 (2): 181-185.

12. Jam R, Mesquida J, Hernández Ó et al. (2018): Nursing workload and compliance with nonpharmacological measures to prevent ventilatorassociated pneumonia: a multicentre study. Nursing in Critical Care, 23(6): 291-298.

13. Metersky M, Wang $Y$, Klompas $M$ et al. (2016): Trend in ventilator-associated pneumonia rates between 2005 and 2013. JAMA., 316 (22): 2427-2429.

14. Gamberini L, Giugni A, Ranieri S et al. (2019): Early-onset ventilator-associated pneumonia in severe traumatic brain injury: is there a relationship with prehospital airway management? The Journal of Emergency Medicine, 56 (6): 657-665.

15. Su K, Kou Y, Lin F et al. (2017): A simplified prevention bundle with dual hand hygiene audit reduces early-onset ventilator-associated pneumonia in cardiovascular surgery units: An interrupted time-series analysis. PloS One, 12 (8): 252-56.

16. Rabello F, Araújo V, Magalhães S (2018): Effectiveness of oral chlorhexidine for the prevention of nosocomial pneumonia and ventilator- associated pneumonia in intensive care units: Overview of systematic reviews. International Journal of Dental Hygiene, 16 (4): 441-449.

17. Lau A, So H, Tang S et al. (2015): Prevention of ventilator-associated pneumonia. Hong Kong Med J., 21 (1): 61-68.

18. Waters B, Muscedere J (2015): A 2015 update on ventilator-associated pneumonia: new insights on its prevention, diagnosis, and treatment. Current Infectious Disease Reports, 17 (8): 1-9.

19. Othman A, Abdelazim M (2017): Ventilatorassociated pneumonia in adult intensive care unit prevalence and complications. The Egyptian Journal of Critical Care Medicine, 5 (2): 61-63.

20. Thatrimontrichai $\mathrm{A}$, Techato $\mathrm{C}$, Dissaneevate $\mathrm{S}$ et al. (2016): Risk factors and outcomes of carbapenemresistant Acinetobacter baumannii ventilator-associated pneumonia in the neonate: A case-case-control study. Journal of Infection and Chemotherapy, 22 (7): 444-449. 